\title{
Refractory Waldenstrom Macroglobulinemia
}

National Cancer Institute

\section{Source}

National Cancer Institute. Refractory Waldenstrom Macroglobulinemia. NCI Thesaurus.

Code C142149.

Waldenstrom macroglobulinemia that does not respond to treatment. 